\title{
Association between sedentary behavior and the risk of dementia: a systematic review and meta-analysis
}

\author{
Shijiao Yan', Wenning Fu², Chao Wang ${ }^{3}$, Jing Mao ${ }^{2}$, Bing Liư ${ }^{4}$, Li Zou ${ }^{5}$ and Chuanzhu Lv 6,7,8
}

\begin{abstract}
An increasing number of original studies suggest that sedentary behavior is associated with the risk of dementia, but the results remain inconsistent and inconclusive. In this meta-analysis, we analyzed available observational epidemiological evidence to identify the association between sedentary behavior and the risk of dementia. We searched PubMed and Embase from their inception to March 2019 to identify observational studies examining the association between sedentary behavior and risk of dementia. Two authors independently extracted data and assessed study quality using predefined criteria. The $Q$ statistics and $I^{2}$ methods were used to test for heterogeneity. The publication bias of the included studies was also estimated using Begg's and Egger's tests. We identified 18 relevant cohort studies involving 250,063 participants and 2269 patients with dementia. Pooled result showed that sedentary behavior was significantly associated with increased risk of dementia ( $R R=1.30 ; 95 \%$ Cl: 1.12-1.51). In addition, subgroup analyses by state, and controlling for the concomitant effects of age, sex, education were conducted for the increase of dementia risk, relating to sedentary, respectively. In general, these subgroup analyses showed no statistically significant differences. The results of our meta-analysis suggested that sedentary behavior was independently associated with a significantly increased risk of dementia, which might have important implications in conducting etiological studies for dementia and developing strategies for dementia prevention.
\end{abstract}

\section{Introduction}

With the consistent improvement in living standards, human life expectancy has increased. Some studies have shown that the prevalence of dementia is also appears to be gradually increasing ${ }^{1,2}$. Dementia is a complex neurological disorder with an irreversible and nonlinear development process. Dementia does not have only a variety of complex causes, but also will brings with many other health problems. Currently, dementia is the fourth leading cause of death following cancer, heart disease, and

\footnotetext{
Correspondence: Li Zou (zouli1231@163.com) or

Chuanzhu Lv (Ivchuanzhu677@126.com)

${ }^{1}$ School of International Education, Hainan Medical University, Haikou, Hainan, China

${ }^{2}$ School of Nursing, Tongji Medical College, Huazhong University of Science and Technology, Wuhan, Hubei, China

Full list of author information is available at the end of the article

These authors contributed equally: Shijiao Yan, Wenning Fu
}

cerebrovascular disease in the elderly ${ }^{3,4}$. According to the International Association of Alzheimer's Disease International Statistics Annual Report 2011, 36 million people worldwide currently experience dementia, and the incidence is increasing at a rate of 1 patient every $7 \mathrm{~s}$. It is estimated that the number of individuals experiencing senile dementia will increase to 66 million by 2030 and reach 115 million by $2050^{5}$. Studies have shown that reducing the modifiable risk factors of dementia may contribute to the prevention and control of dementia ${ }^{6,7}$; hence, identifying its possible risk factors is significantly important.

In recent years, there has been a growing interest in physical exercise as a non-pharmacological treatment for dementia $^{8,9}$. Certainly, some studies have reported that regular physical exercise can favorably affect the physical and cognitive functions of patients with dementia ${ }^{10,11}$. 
Conversely, physical inactivity and a sedentary lifestyle are considered the risk factors of dementia. Studies assessing the association between sedentary behavior and the occurrence of dementia have increased in recent years. However, until now, there have been no consistent conclusions regarding the association between sedentary behavior and the risk of dementia. To the best of our knowledge, the risk of dementia and sedentary lifestyle has not yet been evaluated by a meta-analysis, which is a generally accepted statistical tool for combining results to produce a more precise estimation of associations in different studies ${ }^{12,13}$. Therefore, we conducted a metaanalysis of 18 cohort studies to evaluate the association between sedentary behavior and the risk of dementia. Considering the significant health and economic burden of dementia, the results of our study may provide additional practical and valuable treatments for dementia prevention.

\section{Materials and methods}

Approval by institutional review boards is not required for this systematic review of previously published deidentified data.

\section{Literature search strategy}

We conducted this meta-analysis in accordance with the Preferred Reporting Items for Systematic Reviews and Meta-Analyses ${ }^{14}$ and the checklist of items in the MetaAnalysis Of Observational Studies in Epidemiology ${ }^{15}$. We performed a comprehensive search on PubMed, Embase, and Web of Science databases from their inception to March 2018 for cohort studies published in peer-reviewed journals describing an association between sedentary behavior and the risk of dementia. We used the following keywords to identify relevant citations: "sedentary" or "sedentariness" or "long time sitting" in combination with "dementia" or "Alzheimer's disease" or "AD" or "cogniti". Only articles published in the English language were considered. In addition, reference lists of the retrieved original articles and relevant review articles were also comprehensively examined to identify further pertinent studies.

\section{Study selection}

Studies meeting the following criteria were included in the meta-analysis: (1) the study design was cohort; (2) sedentary behavior was the exposure variable and the outcome was the incidence of dementia; (3) the study reported the relative risks (RRs) with the corresponding 95\% confidence intervals (CIs) of dementia associated with sedentary behavior or included the data needed for their calculation. Animal studies, clinical trials, reviews, letters, and commentaries were excluded. Only studies with detailed information on both sedentary behavior and the incidence of dementia were included.

\section{Data extraction and quality assessment}

Two authors (S.Y. and W.F.) independently extracted data in a standardized fashion. The following data were included: first author, publication year, country (state), age, sample size, cases, adjusted factors, and adjusted RR with $95 \%$ CI. Discrepancies were resolved by a discussion with a third author (L.Z.). Adjusted RRs were selected over the unadjusted risk estimates. In cases where multiple risk estimates were reported in the same study, for example, when the study introduced risk estimates for 3 different years, those risk estimates were included as separate risk estimates.

Quality assessment was performed according to the Newcastle-Ottawa quality assessment scale ${ }^{16}$, which is a validated scale for nonrandomized studies in metaanalyses. This scale assigns a maximum of nine points to each study: four for the selection of participants and measurement of exposure, two for comparability of cohorts or cases and controls based on the design or analysis, and three for the assessment of outcomes and adequacy of follow-up. We assigned scores of $0-3,3.5-6$, and 6.5-9 for low-, moderate-, and high-quality studies, respectively. When the studies had several adjustment models, we extracted those that reflected the maximum extent of adjustment for potentially confounding variables. Disagreement between the two authors' independent data analysis was resolved through a review by the third author.

\section{Statistical analyses}

The $R R$ was considered as the common measure of the association between sedentary behavior and the risk of dementia. When $R R s$ were reported separately for subgroups by the different sedentary times in one study, the fixed-effects model was used to combine the results of the subgroups and calculate a common $R R$ for the main analysis $^{12}$. In addition, the random-effects model was used to calculate an overall pooled $R R$ for the main analysis.

Heterogeneity was identified according to Cochran's $Q$ test, with $P<0.10$ indicating heterogeneity. The $I^{2}$ statistics measured the percentage of total variation through studies due to heterogeneity rather than chance. It was calculated according to the formula by Higgins ${ }^{17} \cdot I^{2}$ was used to quantify the heterogeneity, with $25 \%, 50 \%$, and $75 \%$ indicating low, moderate, and high degrees of heterogeneity, respectively.

Subgroup analyses were performed to determine the possible influence of factors such as state or region and weather. The concomitant effects of age, sex, and educational level were controlled in some studies. The Begg's rank correlation and the Egger's linear regression tests 
were used to assess potential publication bias ${ }^{18,19}$. Moreover, the Duval and Tweedie's nonparametric trim-and-fill method were used to adjust potential publication bias ${ }^{20}$. All analyses were performed using the Stata statistical software (version 12.0; College Station, TX, USA), and all tests were two-sided with a significance level of 0.05 .

\section{Results}

\section{Literature search}

A total of 3617 articles were searched from the electronic databases, and 2215 articles were removed because of duplication. Another 1402 unqualified records were excluded after browsing the titles or abstracts. Among these, 1269 papers reported irrelevant studies, which did not analyze the association between sedentary behavior and the risk of developing dementia, 88 were non-cohort studies, and 27 articles offered insufficient information. After retrieving and reviewing the full text, we determined that 18 cohort studies met our inclusion criteria ${ }^{21-38}$. The study selection procedure is presented in the flow chart in Fig. 1.

\section{Characteristics of the included studies}

Table 1 shows the main characteristics of the 18 cohort studies included in the systematic review. The 18 cohort studies were published between 1984 and 2018. Most of these studies (11) were conducted in Europe $(R R=1.37$, 95\% $\mathrm{CI}=1.14-1.65)$, four in America $(R R=1.52,95 \%$ $\mathrm{CI}=1.30-1.77)$, two in Asia $(R R=0.76,95 \% \mathrm{CI}=$

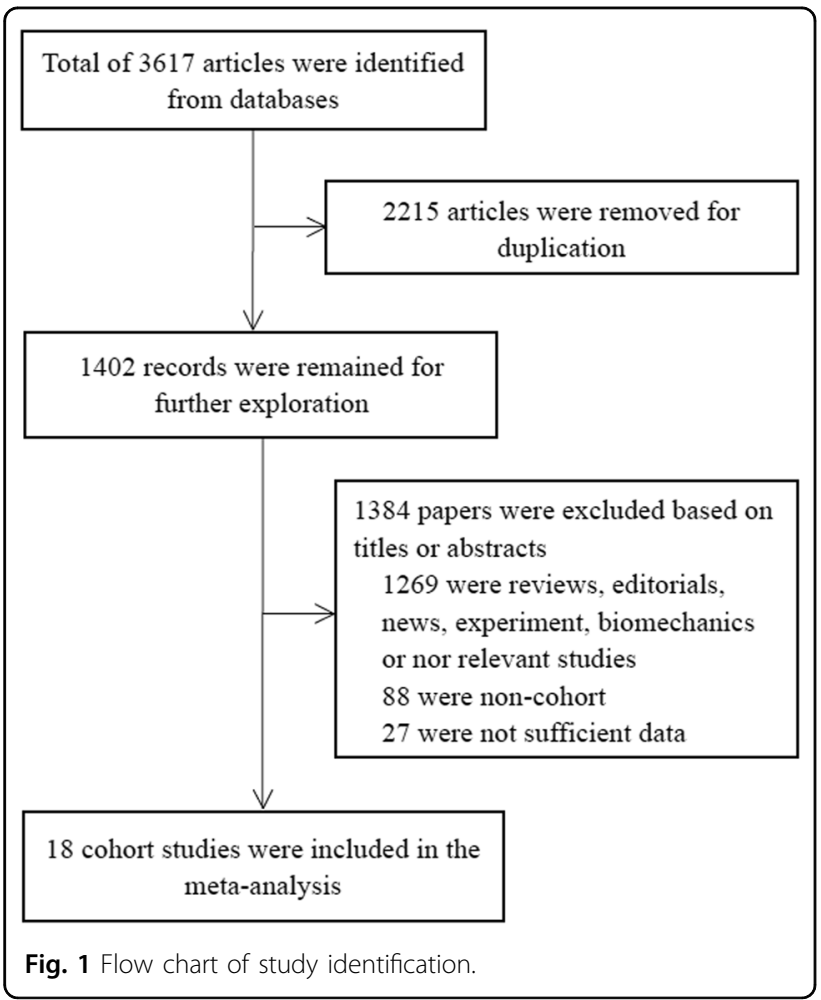

$0.52-1.11)$, and one in Australia $(R R=0.98,95 \% \mathrm{CI}=$ $0.89-1.08)$. The sample size of these studies ranged from 33 to 490 with a total of 250,063 participants. Seventeen studies were published after 2000 , and only one study was published before 2000. The quality assessment scores ranged from 6 to 9, with an average score of 7 points, representing satisfactory quality of the studies.

\section{Results of meta-analysis \\ Association between sedentary behavior and the risk of dementia}

Figure 2 shows the results from the random-effects model combining the $R R \mathrm{~s}$ for dementia in relation to sedentary behavior. Eighteen studies investigated the association between sedentary behavior and risk of dementia. The pooled $R R$ of dementia for sedentary behavior was 1.30 (95\% CI, 1.12-1.51), with a substantial heterogeneity across studies $\left(P=0.000, I^{2}=66.9 \%\right)$.

\section{Results of subgroup and sensitivity analyses Subgroup analyses}

Table 2 shows the results of subgroup analyses. To assess the stability of the primary estimates outcomes and identify the potential resources of heterogeneity, we performed subgroup analyses. Subgroup analyses by state, controlling for the concomitant effects of age, sex, and educational level, were performed to assess the increased risk of dementia as a result of a sedentary behavior. In general, these subgroup analyses showed no statistically significant difference since the pooled results of all subgroups showed a positive and statistically significant association between sedentary behavior and the risk of dementia.

\section{Sensitivity analyses}

We performed sensitivity analyses to determine the potential sources of heterogeneity in the association between sedentary behavior and risk of dementia, examine the influence of various exclusions on the combined $R R$, and assess the robustness of all results. We compared the fixed-effect and random-effect models, but found no significant difference in the pooled RRs between the two

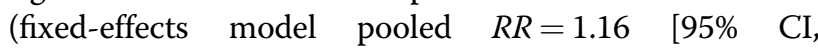
$1.09-1.24]$, random-effects model pooled $R R=1.30$ [95\% CI, 1.12-1.51]). In addition, each study was excluded in turn, and the results of the remaining studies were pooled together. The pooled RR did not significantly change, ranging from 1.27 (95\% CI, $1.10-1.47)$ to 1.34 (95\% CI, $1.16-1.56)$, and the heterogeneity was detected with an $I^{2}=65.4 \%$ and $66.3 \%$, respectively.

\section{Publication bias}

Visual inspection of the funnel plot showed significant asymmetry (Fig. 3). The Egger and Begg test indicated no 


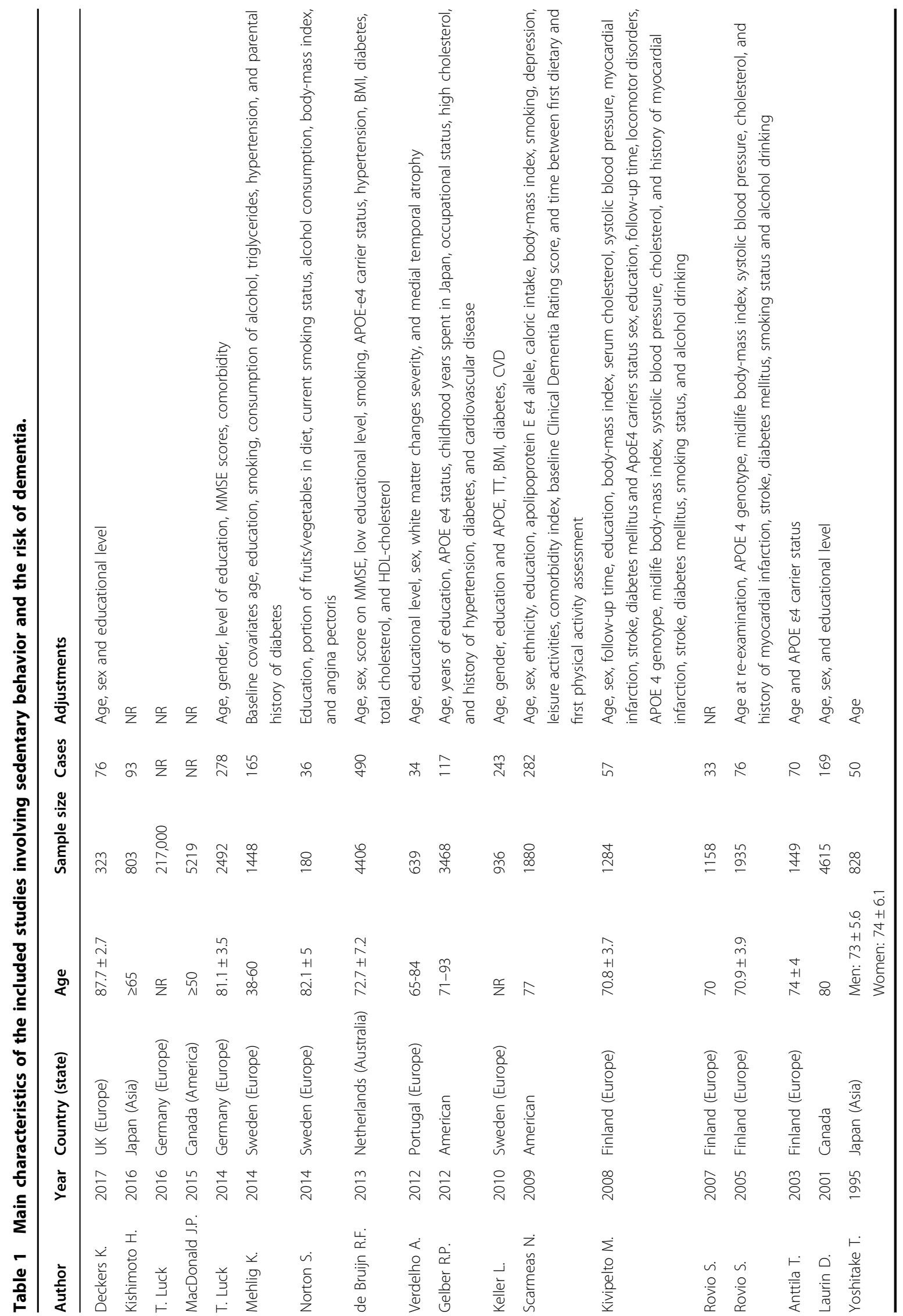


evidence of publication bias (Egger, $P=0.677$; Begg, $P=$ 0.074). The trim-and-fill method was used to evaluate the impact of any potential publication bias, and the results showed that two potentially missing studies would be required to obtain the funnel plot symmetry for the association between sedentary behavior and the risk of dementia (Fig. 4). After using the trim-and-fill method, the corrected $R R$ was 1.27 (95\% CI, 1.10-.47; randomeffects model, $P=0.000$ ), suggesting that the pooled $R R$ did not significantly change by the correction for potential publication bias.

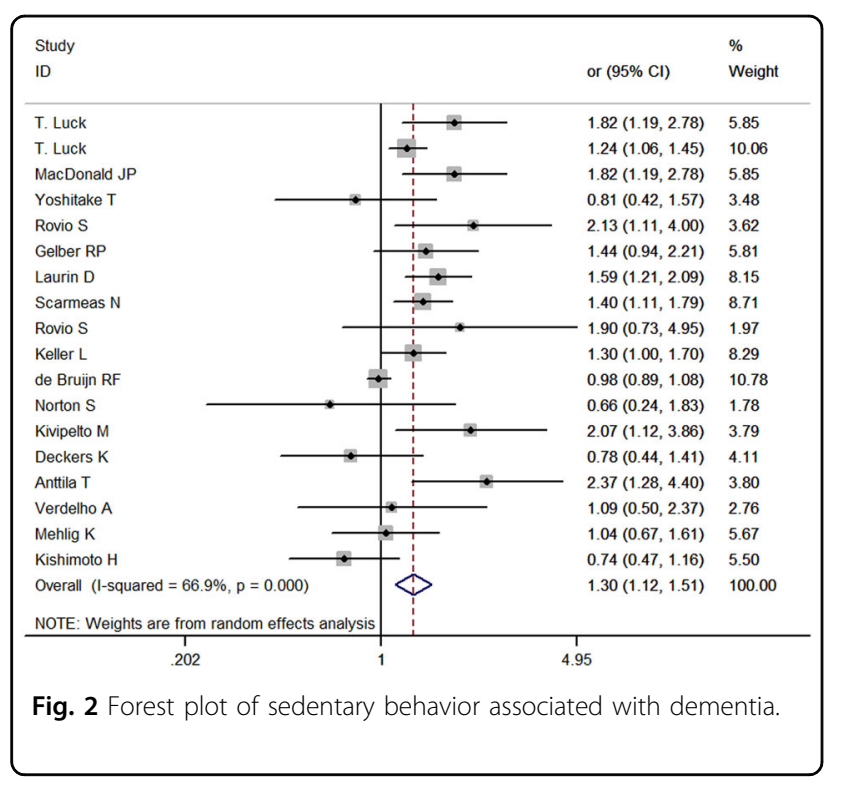

\section{Discussion}

With the rapid development of information technology, people are able to receive essential information without leaving their homes. Artificial intelligence and mobile terminals (such as mobile phones and laptops) enable individuals to interact with other individuals worldwide in a static or sedentary manner. Therefore, sedentary lifestyle has been considered to be significantly prevalent in the current society, specifically for office workers, students, and drivers. Previous reviews have suggested that sedentary behavior was associated with lower cognitive performance, although the attributable risk of sedentary time to all-cause dementia incidence is unclear $^{39,40}$.

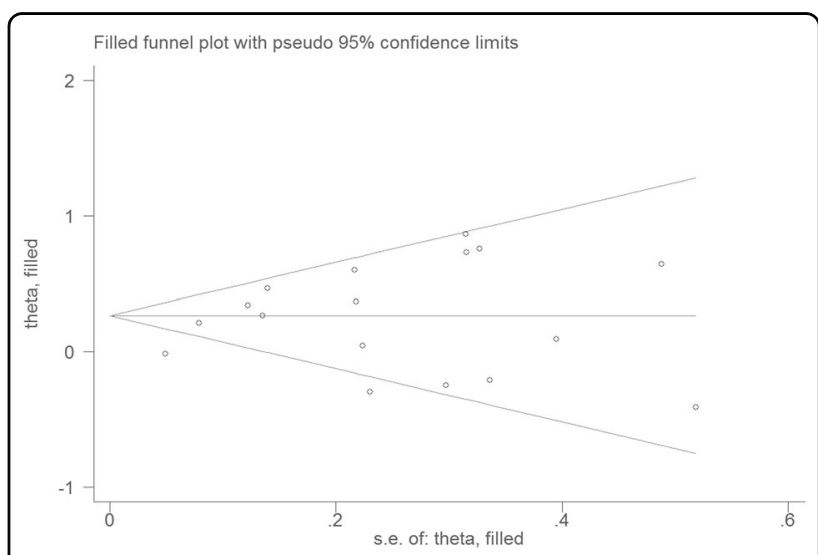

Fig. 3 Funnel plot for studies of sedentary behavior and dementia. The horizontal line represents summary effect estimates, and two slashes lines are pseudo 95\% Cls.

Table 2 Results of subgroup analyses about sedentary behavior and the risk of dementia.

\begin{tabular}{|c|c|c|c|c|c|c|}
\hline Subgroup & Number of studies & $R R$ & $95 \%$ confidence intervals & $P$ for heterogeneity & $I^{2}(\%)$ & $P$ Value for interaction \\
\hline \multicolumn{7}{|l|}{ State } \\
\hline Asia & 2 & 0.76 & $0.52-1.11$ & 0.825 & 0.00 & \multirow[t]{4}{*}{$>0.05$} \\
\hline America & 4 & 1.51 & $1.30-1.77$ & 0.733 & 0.00 & \\
\hline Europe & 11 & 1.37 & $1.14-1.65$ & 0.066 & 42.5 & \\
\hline Australia & 1 & 0.98 & $0.89-1.09$ & - & - & \\
\hline \multicolumn{7}{|c|}{ Controlling for age } \\
\hline Yes & 5 & 1.30 & $0.82-2.06$ & 0.011 & 69.3 & \multirow[t]{2}{*}{$>0.05$} \\
\hline No & 13 & 1.29 & $1.10-1.51$ & 0.000 & 67.2 & \\
\hline \multicolumn{7}{|c|}{ Controlling for sex } \\
\hline Yes & 9 & 1.29 & $1.08-1.54$ & 0.000 & 72.3 & \multirow[t]{2}{*}{$>0.05$} \\
\hline No & 9 & 1.30 & $0.98-1.74$ & 0.011 & 59.8 & \\
\hline \multicolumn{7}{|c|}{ Controlling for education } \\
\hline Yes & 12 & 1.26 & $1.08-1.47$ & 0.001 & 64.8 & \multirow[t]{2}{*}{$>0.05$} \\
\hline No & 6 & 1.42 & $0.95-2.13$ & 0.006 & 69.5 & \\
\hline
\end{tabular}




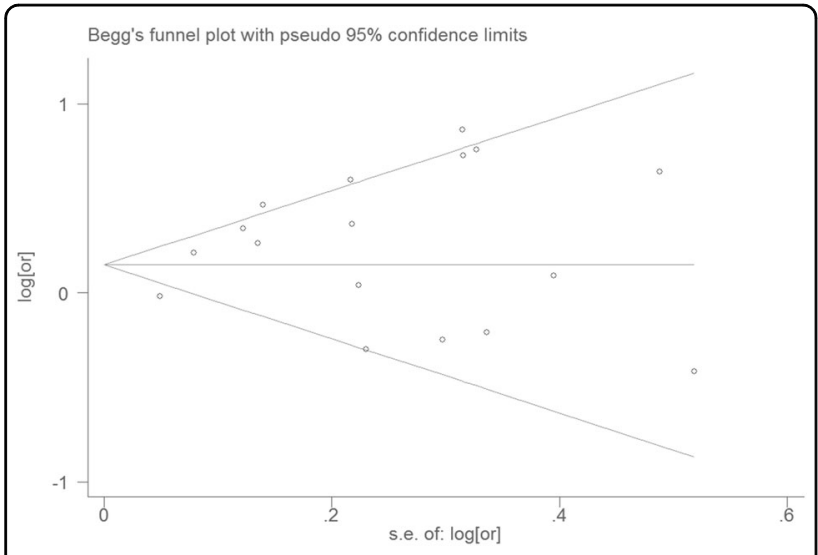

Fig. 4 Filled funnel plot of $R R$ from studies that investigated the association between sedentary behavior and the risk of dementia. The horizontal line represents summary effect estimates, and two slashes lines are pseudo $95 \% \mathrm{Cls}$.

However, the meta-analysis of 18 cohort studies involving 250,063 participants and 2269 patients confirmed the positive association between sedentary behavior and the risk of dementia. Compared with individuals who had not been exposed to sedentary behavior, individuals who were exposed to sedentary behavior had a $30 \%$ higher risk of experiencing dementia. Sedentary behavior was associated with several chronic diseases that were also associated with cognitive impairment and risk of dementia ${ }^{41,42}$. Previous data suggested that prolonged sedentary time could impair glucose and lipid metabolism ${ }^{43-45}$, which were recognized as the risk factors for cognitive decline and all-cause dementia ${ }^{46,47}$. In addition, inflammation was also identified as a potential risk factor for dementia ${ }^{48,49}$. Meanwhile, sedentary behavior might conversely induce or aggravate individual inflammation ${ }^{50-52}$. Finally, exercise was considered a protective factor for dementia ${ }^{53}$. However, physical activity declines with age, while sedentary behavior increases with age ${ }^{54,55}$. Voss and his colleagues found that increases in sedentary behavior and sedentary time were significantly observed in individuals who were about to retire and were continuously observed in adults after the age of 60 years, and individuals aged over 80 years are often involved in sedentary behavior, with an average sedentary time of $9 \mathrm{~h}$ per $\mathrm{day}^{56}$, eliminating the benefits of physical exercises on individual's cognitive health.

Considering that a substantial heterogeneity was observed in the included studies, we further performed a subgroup analysis to determine the potential sources of heterogeneity. Based on the result of the subgroup analyses, we found that the pooled odds ratios of the studies conducted in Asia and Australia showed no statistically significant association between sedentary behavior and the risk of dementia. The probable explanation was that only two studies from Asia and one from Australia were included in this meta-analysis. Their sample sizes were small and lacked representation, thereby potentially leading to bias in the results ${ }^{57}$, indicating the need for additional studies in Asia and Australia to determine significant robust results. When the analysis was stratified to control the concomitant effects of age, sex, and educational level, we found that the summarized results from the original studies without adjusting for age, sex, and educational level showed no statistically significant difference in the association between sedentary behavior and risk of dementia $(P>0.05)$. On the contrary, if the original studies were controlled for age, sex, and educational level, the merged $R R$ s were all statistically significant $(P<0.05)$, although the difference between the pooled results in each subgroup was not statistically significant $(P>0.05)$. Specifically, aging was an independent suggested risk factor for developing dementia ${ }^{58,59}$. Our subgroup analysis suggested that age might be a confounding factor in the association between sedentary lifestyle and risk of dementia, consistent with the results when sex and educational level were adjusted. To generalize the findings, more studies determining the association between age, sex, and educational level and sedentary lifestyle and their synergistic effects on the risk of dementia were required.

To assess the consistency and robustness of the results from the primary analysis and determine the potential sources of heterogeneity in the association between sedentary behavior and the risk of dementia, we performed a sensitivity analysis. However, the pooled results showed only minor changes ranging from 1.27 (95\% CI, $1.10-1.47)$ to 1.34 (95\% CI, 1.16-1.56) after excluding each study in turn from the analysis, highlighting the robustness and reliability of the primary result. On the contrary, publication bias was observed in the included studies, but the primary result did not significantly change after using the "trim-and-fill" method, significantly confirming the robustness of our findings.

This meta-analysis has several strengths. First, the study design of all the studies in our analysis was cohort, which was considered as a stronger measure when demonstrating causation and identifying risk factors than other observational study designs ${ }^{60}$. Second, consistent results from the sensitivity analysis indicated that our findings were reliable and robust, although heterogeneity was observed among the studies. Third, when several RRs were introduced separately in terms of the different sedentary times, we combined the results of these subgroups and calculated a common result using the fixedeffects model. Therefore, we could pool the outcomes regarding the association between the risk of dementia and sedentary behavior with non-sedentary behavior.

On the contrary, this meta-analysis has several limitations that may affect the interpretation of the results in 
our analysis. First, the heterogeneity of the included studies was significant $\left(I^{2}>60 \%\right)$ and was observed throughout the analyses. However, we determined the potential sources of heterogeneity by performing subgroup analyses and sensitivity analysis. Second, considering the limited information provided in original studies, a dose-response analysis was not performed to provide further evidence supporting the association between sedentary behavior and the risk of dementia. Third, the definition of "sedentary behavior" was inconsistent, which might induce bias in effect size estimates. Fourth, the outcome we were interested in was all-cause dementia. Considering that the information related to the subtypes of dementia was insufficient, we did not analyze the effects of sedentary lifestyle on specific dementia subtypes, such as vascular dementia and senile dementia.

\section{Conclusion}

The results of this meta-analysis of cohort studies with the most up-to-date evidence suggested that sedentary behavior was significantly associated with an increased risk of dementia. The positive association remained consistent throughout the analysis. Considering the increasing prevalence of sedentary behavior in modern society, our findings have been significant for policy makers and health education institutions. Considering the insufficient information on the dose-response association of sedentary time and the risk of developing dementia, well-designed studies with adequate analyses of sedentary time and the risk of dementia are required to confirm our findings.

\section{Acknowledgements}

We thank all staff members involved in this study. This study was supported by the Hainan Provincial Science and Technology Major Project (ZDKJ201804).

\begin{abstract}
Author details
'School of International Education, Hainan Medical University, Haikou, Hainan, China. ${ }^{2}$ School of Nursing, Tongji Medical College, Huazhong University of Science and Technology, Wuhan, Hubei, China. ${ }^{3}$ School of Public Health, Tongji Medical College, Huazhong University of Science and Technology, Wuhan, Hubei, China. ${ }^{4}$ Center of Health Administration and Development Studies, Hubei University of Medicine, Shiyan, Hubei, China. ${ }^{5}$ Department of Neurology, Taihe Hospital, Hubei University of Medicine, Hubei, China. ${ }^{6}$ Department of Emergency, Hainan Clinical Research Center for Acute and Critical Diseases, The Second Affiliated Hospital of Hainan Medical University, Haikou, Hainan, China. ${ }^{7}$ Emergency and Trauma College, Hainan Medical University, No. 3 Xueyuan Road, Longhua Zone, Haikou, Hainan, China. ${ }^{8}$ Key Laboratory of Emergency and Trauma of Ministry of Education, Hainan Medical University, Haikou, Hainan, China
\end{abstract}

\section{Author contributions}

C.L. and W.F. had full access to all the data in the study and took responsibility for the integrity of the data and the accuracy of the data analysis. S.Y. and L.Z. independently extracted the related data information. Check the related data information again: S.Y., W.F., C.W., J.M., B.L., L.Z., and C.L. Data analysis and writing articles: S.Y. and W.F. Analysis or interpretation of data: All authors. Critical revision of the paper for important intellectual content: All authors.

\section{Conflict of interest}

The authors declare that they have no conflict of interest.

\section{Publisher's note}

Springer Nature remains neutral with regard to jurisdictional claims in published maps and institutional affiliations.

Received: 24 September 2019 Revised: 12 March 2020 Accepted: 25 March 2020

Published online: 06 July 2020

\section{References}

1. El Tallawy, H. N. et al. Prevalence of dementia in Al Kharga District, New Valley Governorate, Egypt. Neuroepidemiology 38, 130-137 (2012).

2. Deng, J. et al. Prevalence and effect factors of dementia among the community elderly in Chongqing, China. Psychogeriatrics 18, 412-420 (2018).

3. Huang, W.-y., Yang, X., Yang, J.-y. \& Deng, H. Investigation on prevalence of dementia among elderly in urban communities of Guiyang city. Chin. J. Public Health-Shenyang- 23, 0983 (2007).

4. Chen, X., Huang, D. \& Lin, A. Causes and countermeasures study on psychiatric disabled adults in Guangdong province. Chin. J. Rehabilitation Med. 10, 24 (2009).

5. Prince, M., Bryce, R. \& Ferri, C. World Alzheimer Report 2011 - the benefits of early diagnosis and intervention. National Guideline Clearinghouse (2011).

6. Skov, J. N. et al. Objective cognitive impairment and progression to dementia in women: the prospective epidemiological risk factor study. J. Prev. Alzheimer's Dis. 4, 194-200 (2017).

7. Dominguez, J., de Guzman, F., Reandelar, M. Jr \& Thi Phung, T. K. Prevalence of dementia and associated risk factors: a population-based study in the Philippines. J. Alzheimer's Dis. 63, 1065-1073 (2018).

8. Fleiner, T., Dauth, H., Gersie, M., Zijlstra, W. \& Haussermann, P. Structured physical exercise improves neuropsychiatric symptoms in acute dementia care: a hospital-based RCT. Alzheimer's Res. Ther. 9, 68 (2017).

9. Prick, A.-E., De Lange, J., Scherder, E., Twisk, J. \& Pot, A. M. The effects of a multicomponent dyadic intervention with physical exercise on the cognitive functioning of people with dementia: A randomized controlled trial. J. Aging Phys. Act. 25, 539-552 (2017).

10. Lam, F. M. et al. Physical exercise improves strength, balance, mobility, and endurance in people with cognitive impairment and dementia: a systematic review. J. Physiother. 64, 4-15 (2018).

11. Matura, S. F., Carvalho, A. S., Alves, G. \& Pantel, J. Physical exercise for the treatment of neuropsychiatric disturbances in Alzheimer's dementia: possible mechanisms, current evidence and future directions. Curr. Alzheimer Res. 13, 1112-1123 (2016).

12. DerSimonian, R. \& Laird, N. Meta-analysis in clinical trials. Controlled Clin. Trials $\mathbf{7}$, 177-188 (1986).

13. Zeng, X. et al. The methodological quality assessment tools for preclinical and clinical studies, systematic review and meta-analysis, and clinical practice guideline: a systematic review. J. Evid.-based Med. 8, 2-10 (2015).

14. Moher, D., Liberati, A., Tetzlaff, J. \& Altman, D. G. Preferred reporting items for systematic reviews and meta-analyses: the PRISMA statement. Int J. Surg. 8 336-341 (2010).

15. Stroup, D. F. et al. Meta-analysis of observational studies in epidemiology: a proposal for reporting. Jama 283, 2008-2012 (2000).

16. Peterson, J., Welch, V., Losos, M. \& Tugwell, P. The Newcastle-Ottawa scale (NOS) for Assessing the Quality of Nonrandomised Studies in Meta-analyses (Ottawa Hospital Research Institute, Ottawa, 2011).

17. Higgins, J. P., Thompson, S. G., Deeks, J. J. \& Altman, D. G. Measuring inconsistency in meta-analyses. Bmj 327, 557-560 (2003).

18. Begg, C. B. \& Mazumdar, M. Operating characteristics of a rank correlation test for publication bias. Biometrics 1088-1101 (1994).

19. Egger, M., Smith, G. D., Schneider, M. \& Minder, C. Bias in meta-analysis detected by a simple, graphical test. Bmj 315, 629-634 (1997).

20. Duval, S. \& Tweedie, R. Trim and fill: a simple funnel-plot-based method of testing and adjusting for publication bias in meta-analysis. Biometrics $\mathbf{5 6}$, 455-463 (2000).

21. Luck, T. \& Riedel-Heller, S. Prevention of Alzheimer's dementia in Germany: A projection of the possible potential of reducing selected risk factors. Nervenarzt 87, 1194-1200 (2016)

22. Luck, T. et al. Apolipoprotein E epsilon 4 genotype and a physically active lifestyle in late life: analysis of gene-environment interaction for the risk of 
dementia and Alzheimer's disease dementia. Psychological Med. 44, 1319-1329 (2014).

23. Rist, P. M., Marden, J. R.r Capistrant, B. D., Wu, Q. \& Glymour, M. M. Do physical activity, smoking, drinking, or depression modify transitions from cognitive impairment to functional disability? J. Alzheimer's Dis. 44, 1171-1180 (2015).

24. Yoshitake, T. et al. Incidence and risk factors of vascular dementia and Alzheimer's disease in a defined elderly Japanese population: the Hisayama Study. Neurology 45, 1161-1168 (1995).

25. Rovio, S. et al. Leisure-time physical activity at midlife and the risk of dementia and Alzheimer's disease. Lancet Neurol. 4, 705-711 (2005).

26. Gelber, R. P. et al. Lifestyle and the risk of dementia in Japanese-American men. J. Am. Geriatrics Soc. 60, 118-123 (2012).

27. Laurin, D., Verreault, R., Lindsay, J., MacPherson, K. \& Rockwood, K. Physical activity and risk of cognitive impairment and dementia in elderly persons. Arch. Neurol. 58, 498-504 (2001).

28. Norton, S., Matthews, F. E., Barnes, D. E., Yaffe, K. \& Brayne, C. Potential for primary prevention of Alzheimer's disease: an analysis of population-based data. Lancet Neurol. 13, 788-794 (2014).

29. Andel, R. et al. Physical exercise at midlife and risk of dementia three decades later: a population-based study of Swedish twins. J. Gerontol. Ser. A: Biol. Sci. Med. Sci. 63, 62-66 (2008).

30. de Bruijn, R. F. et al. The association between physical activity and dementia in an elderly population: the Rotterdam Study. Eur. J. Epidemiol. 28, 277-283 (2013).

31. Winblad, B. Apolipoprotein E $\varepsilon 4$ magnifies lifestyle risks for dementia: a population-based study. J. Cell Mol. Med. 12, 2762-2771 (2008).

32. Deckers, $\mathrm{K}$. et al. Lack of associations between modifiable risk factors and dementia in the very old: findings from the Cambridge City over-75s cohort study. Aging Ment. Health 22, 1272-1278 (2018).

33. Anttila, T. et al. Midlife income, occupation, APOE status, and dementia: a population-based study. Neurology 59, 887-893 (2002).

34. Verdelho, A. et al. Physical activity prevents progression for cognitive impairment and vascular dementia: results from the LADIS (leukoaraiosis and disability) study. Stroke 43, 3331-3335 (2012).

35. Mehlig, K. et al. Physical activity, weight status, diabetes and dementia: a 34year follow-up of the population study of women in Gothenburg. Neuroepidemiology 42, 252-259 (2014).

36. Kishimoto, $\mathrm{H}$. et al. The long-term association between physical activity and risk of dementia in the community: the Hisayama Study. Eur. J. Epidemiol. 31, 267-274 (2016).

37. Scarmeas, N. et al. Mediterranean diet and mild cognitive impairment. Arch. Neurol. 66, 216-225 (2009).

38. Miklósi, A. Adam Miklósi. Curr. Biol. 21, R973-R974 (2011).

39. Wheeler, M. J. et al. Sedentary behavior as a risk factor for cognitive decline? A focus on the influence of glycemic control in brain health. Alzheimer's Dement: Transl. Res. Clin. Interventions 3, 291-300 (2017).

40. Falck, R. S., Davis, J. C. \& Liu-Ambrose, T. What is the association between sedentary behaviour and cognitive function? A systematic review. Br. J. Sports Med. 51, 800-811 (2017).

41. Owen, N., Healy, G. N., Matthews, C. E. \& Dunstan, D. W. Too much sitting: the population-health science of sedentary behavior. Exerc. Sport Sci. Rev. 38, 105 (2010).
42. González, K., Fuentes, J. \& Márquez, J. L. Physical inactivity, sedentary behavior and chronic diseases. Korean J. Fam. Med. 38, 111 (2017).

43. Duvivier, B. M. et al. Minimal intensity physical activity (standing and walking) of longer duration improves insulin action and plasma lipids more than shorter periods of moderate to vigorous exercise (cycling) in sedentary subjects when energy expenditure is comparable. PLOS ONE 8 (2013).

44. Healy, G. N., Matthews, C. E., Dunstan, D. W., Winkler, E. A. \& Owen, N. Sedentary time and cardio-metabolic biomarkers in US adults: NHANES 2003-06. Eur. Heart J. 32, 590-597 (2011).

45. Tremblay, M. S., Colley, R. C., Saunders, T. J., Healy, G. N. \& Owen, N. Physiological and health implications of a sedentary lifestyle. Appl. Physiol, Nutr., Metab. 35, 725-740 (2010).

46. Messier, C. \& Teutenberg, K. The role of insulin, insulin growth factor, and insulin-degrading enzyme in brain aging and Alzheimer's disease. Neural Plasticity 12, 311-328 (2005).

47. Panza, F. et al. Lipid metabolism in cognitive decline and dementia. Brain Res. Rev. 51, 275-292 (2006).

48. Dregan, A., Chowienczyk, P. \& Gulliford, M. C. Are inflammation and related therapy associated with all-cause dementia in a primary care population? J. Alzheimer's Dis. 46, 1039-1047 (2015).

49. Simone, M. J. \& Tan, Z. S. The role of inflammation in the pathogenesis of delirium and dementia in older adults: a review. CNS Neurosci. Therapeutics 17, 506-513 (2011).

50. Alves, B. C., da Silva, T. R. \& Spritzer, P. M. Sedentary lifestyle and highcarbohydrate intake are associated with low-grade chronic inflammation in post-menopause: a cross-sectional study. Rev. Brasileira de. Ginecologia e Obstetrícia/RBGO Gynecol. Obstet. 38, 317-324 (2016).

51. Edwards, M. K. \& Loprinzi, P. D. Systemic inflammation as a function of the individual and combined associations of sedentary behaviour, physical activity and cardiorespiratory fitness. Clin. Physiol. Funct. Imaging 38, 93-99 (2018).

52. León-Latre, M. et al. Sedentary lifestyle and its relation to cardiovascular risk factors, insulin resistance and inflammatory profile. Rev. Española de. Cardiolía 67, 449-455 (2014).

53. Keenan, B., Jenkins, C. \& Ginesi, L. Preventing and diagnosing dementia. Nursing 112, 22-25 (2016).

54. Evenson, K. R., Buchner, D. M. \& Morland, K. B. Objective measurement of physical activity and sedentary behavior among US adults aged 60 years or older. Prev. Chron. Dis. 9 (2012).

55. Healy, G. N. et al. Measurement of adults' sedentary time in population-based studies. Am. J. Preventive Med. 41, 216-227 (2011).

56. Voss, M. W., Carr, L. J., Clark, R. \& Weng, T. Revenge of the "sit" II: does lifestyle impact neuronal and cognitive health through distinct mechanisms associated with sedentary behavior and physical activity? Ment. Health Phys. Act. 7, 9-24 (2014).

57. Nemes, S., Jonasson, J. M., Genell, A. \& Steineck, G. Bias in odds ratios by logistic regression modelling and sample size. BMC Med. Res. Methodol. 9, 56 (2009).

58. Association As. Alzheimer's disease facts and figures. Alzheimer's Dement. 10, e47-e92 (2014).

59. Hebert, L. et al. Change in risk of Alzheimer disease over time. Neurology $\mathbf{7 5}$ 786-791 (2010).

60. Thiese, M. S. Observational and interventional study design types; an overview. Biochemia Med.: Biochemia Med. 24, 199-210 (2014). 